\title{
Gut Microbiota Composition in Mid-Pregnancy Is Associated with Gestational Weight Gain but Not Prepregnancy Body Mass Index
}

\author{
Anna-Katariina Aatsinki, BM, , Henna-Maria Uusitupa, PhD, Eveliina Munukka, PhD?, \\ Henri Pesonen, DSci (Tech), Anniina Rintala, MSc, ${ }^{2,3}$ Sami Pietilä, MSc, Leo Lahti, DSci (Tech), \\ Erkki Eerola, MD, PhD, Linnea Karlsson, MD, PhD, ${ }^{1,6}$ and Hasse Karlsson, MD, PhD ${ }^{1,7}$
}

\begin{abstract}
Background: Pregnancy is a time of numerous hormonal, metabolic, and immunological changes for both the mother and the fetus. Furthermore, maternal gut microbiota composition (GMC) is altered during pregnancy. One major factor affecting GMC in pregnant and nonpregnant populations is obesity. The aim was to analyze associations between maternal overweight/obesity, as well as gestational weight gain (GWG) and GMC. Moreover, the modifying effect of depression and anxiety symptom scores on weight and GMC were investigated.

Methods: Study included 46 women from the FinnBrain Birth Cohort study, of which 36 were normal weight, and 11 overweight or obese according to their prepregnancy body mass index (BMI). Stool samples were collected in gestational week 24, and the GMC was sequenced with Illumina MiSeq approach. Hierarchical clustering was executed to illuminate group formation according to the GMC. The population was divided according to Firmicutes and Bacteroidetes dominance. Symptoms of depression, general anxiety, and pregnancy-related anxiety were measured by using standardized questionnaires.

Results: Excessive GWG was associated with distinct GMC in mid-pregnancy as measured by hierarchical clustering and grouping according to Firmicutes or Bacteroidetes dominance, with Bacteroidetes being prominent and Firmicutes being less prominent in the GMC among those with increased GWG. Reduced alpha diversity was observed among the Bacteroidetes-dominated subjects. There were no zero-order effects between the abundances of bacterial genera or phyla, alpha or beta diversity, and prepregnancy BMI or GWG.

Conclusion: Bacteroidetes-dominated GMC in mid-pregnancy is associated with increased GWG and reduced alpha diversity.
\end{abstract}

Keywords: gut microbiota, pregnancy, obesity, weight gain, psychological stress

\section{Introduction}

$\mathbf{O}$ BESITY IS AN increasing health problem especially in the Western world. In Finland, as many as 35\% of pregnant women are overweight, ${ }^{1}$ and the trend is worldwide. ${ }^{2,3}$ Obesity predisposes mothers-to-be to various pregnancy complications, such as reduced insulin sensitivity, potentially followed by metabolic inflammation and gestational diabetes mellitus. ${ }^{4,5}$ In addition, increased rates of pre-eclampsia, cesarean section, and preterm birth have been reported among obese women in comparison with their normal-weight counterparts. ${ }^{6}$

Furthermore, maternal obesity also affects infant outcomes, including congenital anomalies in the central nervous system and heart, as well as a higher fat percentage later in adolescence,

\footnotetext{
${ }^{1}$ The FinnBrain Birth Cohort Study, Turku Brain and Mind Center, Institute of Clinical Medicine, University of Turku, Turku, Finland. ${ }^{2}$ Department of Medical Microbiology and Immunology, Institute of Biomedicine, University of Turku, Turku, Finland.

${ }^{3}$ Department of Clinical Microbiology and Immunology, Turku University Hospital and University of Turku, Turku, Finland.

${ }^{4}$ Bioinformatics, Turku Center for Biotechnology, Turku, Finland.

${ }^{5}$ Department of Mathematics and Statistics, University of Turku, Turku, Finland.

Departments of ${ }^{6}$ Child Psychiatry and ${ }^{7}$ Psychiatry, Turku University Hospital and University of Turku, Turku, Finland.
}

(C) Anna-Katariina Aatsinki et al. 2018; Published by Mary Ann Liebert, Inc. This Open Access article is distributed under the terms of the Creative Commons Attribution Noncommercial License (http://creativecommons.org/licenses/by-nc/4.0/) which permits any noncommercial use, distribution, and reproduction in any medium, provided the original author(s) and the source are cited. 
and an increased risk for asthma and neuropsychiatric symptoms. ${ }^{6-9}$ In a similar manner, maternal gestational weight gain (GWG) above normal has been reported to serve as a potential risk factor for childhood obesity in the offspring. ${ }^{10}$

The human gastrointestinal tract is inhabited by a vast number of commensal microorganisms that are collectively referred to as gut microbiota (GM). ${ }^{11}$ This densely populated microbial ecosystem is a central component that affects host physiology and metabolism. ${ }^{11} \mathrm{GM}$ contributes to the host's energy harvest from ingested nutrients by hydrolyzing and fermenting complex polysaccharides in the colon. ${ }^{11}$ The high diversity of these bacterial communities has been suggested to serve as a signature of a healthy gut ecosystem ${ }^{12}$ and on the other hand reduced bacterial diversity has been associated, for example, with obesity, ${ }^{13}$ inflammatory bowel disease (IBD) ${ }^{14}$ and a higher amount of chromogranin A, which is a marker of neuroendocrine activation. ${ }^{15}$ Thus, altered and somewhat distorted gut microbiota composition (GMC) may mediate the health implications of metabolic conditions such as obesity. ${ }^{16}$

Previously, certain shifts in GMC have been reported in obesity both in human and rodent studies. ${ }^{17-20}$ Fecal microbiota transplantation (FMT) studies conducted in germ-free mice have demonstrated that, despite a low-fat diet, transplantation from obese individuals causes greater fat accumulation in recipients compared with the mice receiving stools from lean phenotypes. ${ }^{21}$ In a human FMT study, improved insulin sensitivity, but no change in weight, was observed in obese patients with metabolic disturbances after stool transplantation from lean donors. ${ }^{22}$

Obese individuals seem to have a higher proportion of the Gram-positive Firmicutes phylum bacteria in their gut compared with their lean counterparts. ${ }^{18,23}$ Additionally, several bacterial genera such as Parabacteroides, Ruminococcus, Campylobacter, Dialister, Porphyromonas, and Staphylococcus are associated with the obese phenotype. ${ }^{23}$ Maternal overweight has been previously linked, for example, to the GMC that contains higher abundance of bacterial species, such as Staphylococcus aureus, Escherichia coli, and other members of the family Enterobacteriacaea, and lower counts of species such as Bifidobacterium longum and Bacteroides fragilis compared with lean. ${ }^{24,25}$

In addition, previous studies have reported complex results concerning the association between weight and proportion of the Gram-negative B. fragilis in expecting mothers. B. fragilis was reported to be more abundant in gestational week (gwk) 24 in lean mothers, ${ }^{24}$ but on the other hand, another study reported it to be more abundant in the first and third trimester in obese mothers compared with lean controls. ${ }^{25}$ However, in these studies, GM analyses were performed by fluorescent in situ hybridization coupled with flow cytometry (FCM-FISH) and quantitative real-time polymerase chain reaction (qPCR).

Normal GWG has been associated with greater abundance of B. fragilis in gwk $24,{ }^{24}$ but excessive GWG has been associated with greater abundance of Bacteroides/Prevotella group. ${ }^{25}$ Moreover, excessive GWG has been associated with the higher fecal numbers of $E$. coli and prevalence of the members in the Clostridium leptum subgroup and Staphylococcus. $^{24}$ Additionally, women with normal weight gain during pregnancy had increased numbers of Bifidobacterium and Akkermansia muciniphila in their gut in comparison with subjects with excessive weight gain. ${ }^{24}$ Furthermore, Koren et al. reported a reduced overall diversity of GM from the first to the third trimester and signs of increased insulin sensitivity and inflammation in pregnant women, which indicates shifts in GMC during pregnancy despite overweight. ${ }^{26}$

Obesity and mood disorders are highly likely to co-occur: obesity increases the risk of depression and vice versa. ${ }^{27}$ This comorbidity may be due to the high prevalence of both diseases, ${ }^{28,29}$ drug therapy side effects, ${ }^{30}$ or shared inherited factors, ${ }^{31}$ however, the overlapping is still poorly understood. Additionally, altered GMC have been reported among patients with major depressive disorders. ${ }^{32}$ Due to co-occurrence, it is relevant to investigate these spectrums of symptoms together.

We used three approaches to investigate the connection of obesity, GWG, and GMC. First, the association between GMC and clinical markers was analyzed in the conventional way. Second, we used unsupervised data-driven statistical methods to cluster the population in two groups according to their GMC in genera level and analyzed the differences with clinical parameters. Third, we divided the study population into two groups dominated either by phylum Bacteroidetes or Firmicutes to compare the effects observed in unsupervised hierarchical clustering and further investigate the differences between the clinical parameters.

The aim of this study was to investigate the associations among the maternal body mass index (BMI), GWG, and GMC, and diversity in mid-pregnancy. We hypothesize that higher maternal BMI and elevated GWG are associated with distorted GMC and reduced diversity.

\section{Materials and methods}

\section{Subjects, weight measurements, questionnaire data}

Subjects were recruited from a larger study (FinnBrain Birth Cohort study; www.finnbrain.fi) conducted in the southwest Finland. The study has been approved by the Joint Ethics Committee of the Hospital District of Southwest Finland and University of Turku. Subjects were contacted at gwk 22 by phone or e-mail. Fifty-two (65\%) pregnant women agreed to participate, and 28 (35\%) declined. The weight gain during pregnancy and self-reported prepregnancy weight were collected from the maternity welfare clinic documents. Subjects were weighed at least four times during the pregnancy and weight gain was classified based on the recommendations of the Institution of Medicine. ${ }^{3}$ The guideline value for the GWG of mothers with normal weight is 11.5$16 \mathrm{~kg}$ and of overweight or obese mothers $7.0-11.5 \mathrm{~kg} .^{33}$ Mothers were classified according to their prepregnancy BMI into three groups, obese $\geq 30.0 \mathrm{~kg} / \mathrm{m}^{2}$, overweight $25.0-30$. $0 \mathrm{~kg} / \mathrm{m}^{2}$, and normal weight $<25.0 \mathrm{~kg} / \mathrm{m}^{2}$.

Information about maternal pre-eclampsia, pregnancyinduced hypertension, and gestational diabetes as well as neonatal measures and outcomes (mode of delivery, birth weight and height, gwks, and neonatal death) was collected from the National Birth Registry provided by the National Institute for Health and Welfare (www.thl.fi). Information about other medical conditions and medications was collected by means of self-reported questionnaires throughout the pregnancy.

All subjects $(n=52)$ were Caucasian females. None of the subjects reported inflammatory gastrointestinal disorders, such as IBD or type 1 or 2 diabetes. One subject, who had an early neonatal death and antimicrobial treatment, was 
excluded from the study and one sample was excluded due to low stool sample quality. Moreover, three subjects reported antibiotic treatment during the previous 2 months before the sample collection and they were excluded. One subject reported celiac disease and was excluded from the study due to recent knowledge that celiac disease has a major impact on GMC. ${ }^{34}$ Thus, altogether 46 subjects were included in the final analyses.

Prenatal maternal psychiatric symptoms were taken into account as a confounding variable in the statistical analyses. Symptoms were analyzed using self-reported questionnaires, including Pregnancy-Related Anxiety Questionnaire-Revised 2 (PRAQ-R2; total score 0-40), ${ }^{35}$ Edinburgh Postnatal Depression Scale (EPDS) (total score 0-30), ${ }^{36}$ and Symptom Checklist-90, anxiety subscale (SCL-90; total score 0-50) ${ }^{37}$ at gwk 24. Data on delivery, including mode of delivery, gestational age and weight, height, and sex of the newborn was obtained from the hospital records. Information on antibiotic treatments during the preceding 2 months, type 1 and 2 diabetes, celiac disease, and IBDs were self-reported. The season of when the sample collection took place was taken into account as a confounding variable, as seasonal variation in GMC has previously been reported at the population level. ${ }^{38}$ The seasons of the sampling time was divided into winter (December to February), spring (March to May), summer (June to August), and autumn (September to November).

\section{Fecal sample collection and next-generation sequencing analysis of GMC}

Subjects self-collected the stool samples at gwk 24 at their homes in sterile collection tubes according to the oral and written instructions. The samples were stored immediately at $-20^{\circ} \mathrm{C}$ after the collection and brought to the laboratory on ice within 24 hours, after which the samples were stored at $-75^{\circ} \mathrm{C}$ for further analysis. Total DNA from the gently thawed fecal samples was extracted with GTX Stool Extraction Kit VER 2.0 and GenoXtract machine (Hain Lifescience, Nehren, Germany) as previously described. ${ }^{39}$ Before the extraction, the samples were mechanically homogenized with MO BIO PowerLyzer 24 Bench Top Bead-Based Homogenizer (MO BIO Laboratories) in $1.4 \mathrm{~mm}$ Ceramic Bead Tubes (MO BIO Laboratories), to enhance the cell lysis. The extractions were performed according to the manufacturer's instructions. The DNA concentrations were measured fluorometrically with the Qubit 2.0 dsDNA HS Assay Kit (Life Technologies), after which the DNAs were stored at $-75^{\circ} \mathrm{C}$.

The microbial profiles of the DNA extracts were analyzed with Illumina MiSeq $16 \mathrm{~S}$ ribosomal RNA (rRNA) genesequencing protocol as previously described. ${ }^{39,40}$ Briefly, an in-house protocol targeting the V4-V5 regions of the bacterial 16S rRNA gene was utilized in the analysis. The V4-V5 region was amplified using the HiFi PCR Kit (KAPA Biosystems, Wilmington, MA) with in-house-generated, indexed primers, modified from Kozich et al. ${ }^{41}$ The forward and reverse primer sequences for the V4-V5 rRNA gene library were: fwd 5'-AATGATACGGCGACCACCGAGATCT ACAC-i5 ${ }_{-}^{a}$ TATGGTAATTGTGTGCCAGCMGCCGCGG TAA-3' and rev 5'-CAAGCAGAAGACGGCATACGAG AT-i $7^{a}$-AGTCAGTCAGGCCCCGTCAATTCMTTTRAGT- $3^{\prime}$, where i5 and i7 represent index sequences (eight nucleotides) enabling the identification of sequences originating from each prespecified DNA sample. The detailed information about the contents of the reactions and condition of the PCR have been described previously. ${ }^{40}$ The PCR products were purified with Agencourt AMPure XP magnetic beads (Beckman Coulter, Brea, CA) on the DynaMag-96 Side Magnet (Thermo Fisher Scientific), and their qualities were controlled by measurement with TapeStation (Agilent Technologies, Santa Clara, CA). Finally, the DNA concentrations of the purified products were measured with the Qubit 2.0 dsDNA HS Assay Kit (Life Technologies).

Raw reads across the 46 samples, sequenced with the Illumina MiSeq 300 bp paired-end sequencing, were used as an input for the data analysis that was performed as previously described. ${ }^{40}$ Reads were first quality filtered requiring at least a 20 Phred quality score, resulting in 51-428k reads per sample (mean: $103 \mathrm{k}$, standard deviation: $52 \mathrm{k}$ ). At the species level, $26 \%$ of the reads was identified as specific species, and at the genus level, $62 \%$ of reads was identified as specific genera. Due to the uncertainty of the species-level results, only genus and higher taxonomic levels were used for the analysis.

\section{Statistical analyses}

The statistical analyses were performed by using R 3.3.3. ${ }^{42}$ The subjects were divided into the two distinct subgroups based on the relative abundances of their bacterial genera. The grouping was performed using the unsupervised hierarchical complete-linkage clustering based on the Bray-Curtis dissimilarities. The identified clusters were characterized by notable tradeoff in the Firmicutes and Bacteroidetes dominance. The Firmicutes-Bacteroidetes ratio (the ratio between their relative abundance in each sample) was used to create groups dominated by Bacteroidetes (Firmicutes/Bacteroidetes ratio $<1$ ) or Firmicutes (Firmicutes/Bacteroidetes ratio $\geq 1$ ).

Alpha diversity indices (Shannon index, Chao1, and number of observed species) were calculated with the $\mathrm{R}$ vegan package. ${ }^{43}$ Beta diversity was calculated with unweighted and weighted UniFrac and the regression models were calculated with the adonis function from $\mathrm{R}$ vegan package as implemented in QIIME bioinformatics software.

Furthermore, the subject phenotypes were investigated based on the available clinical data (BMI, weight gain, questionnaire data) within the clusters. The statistical significance of the difference in distribution of categorical variables in the clusters was determined using Fisher's exact test as the number of subjects was modest. The difference in continuous variables was tested using the Wilcoxon's rank-sum test. The statistical relationships of two continuous variables, including Operational taxonomic unit (OTU) counts and relative proportions of bacteria, BMI and GWG, were investigated with Spearman's rho. $p$-Values were adjusted for multiple testing using Benjamini and Hochberg method. ${ }^{44}$

\section{Results}

\section{Clinical characteristics of the study subjects}

The characteristics of the study subjects $(n=46)$ regarding maternal BMI, GWG, season of sample taking, psychiatric symptoms, medical conditions, and neonatal measures and outcomes are described in Table 1. There was no correlation 


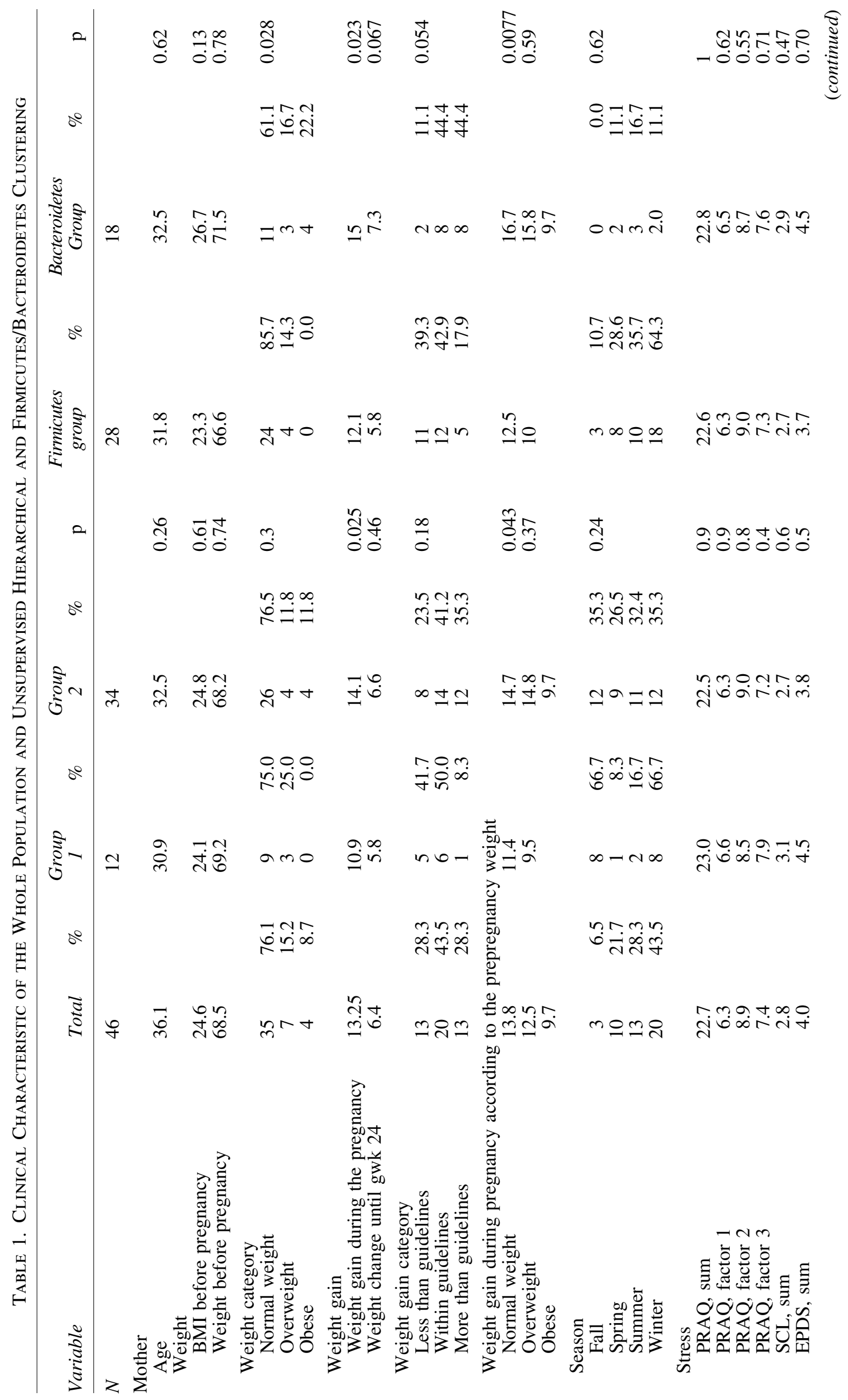




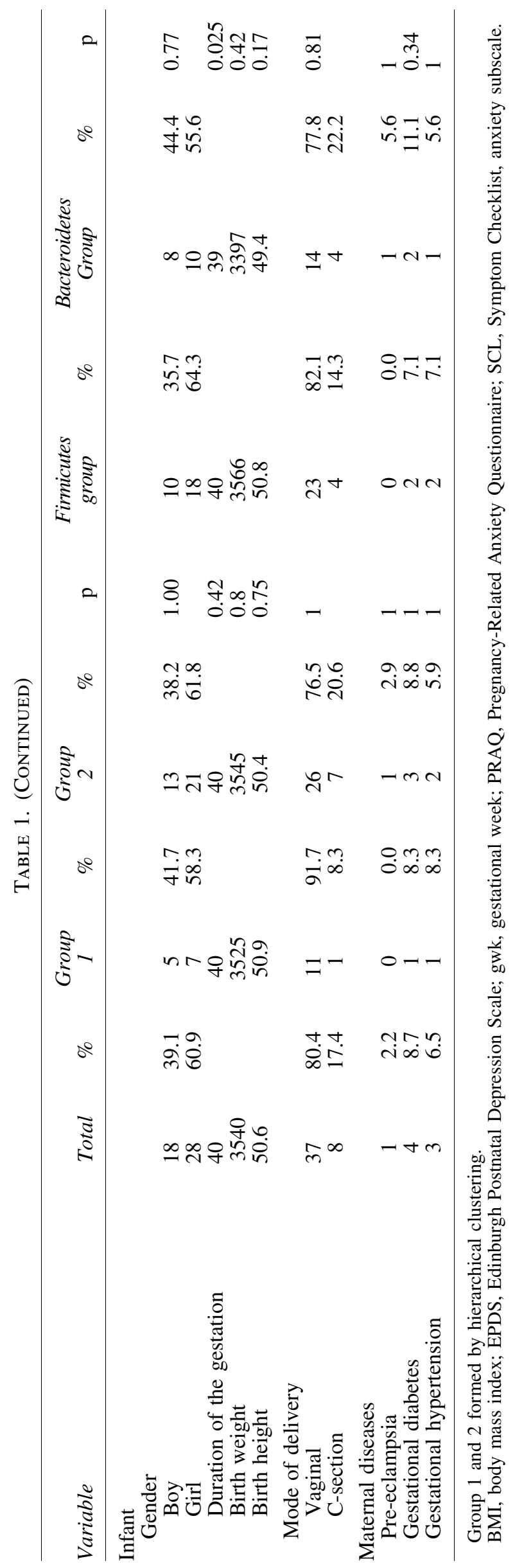


between prepregnancy BMI and GWG during pregnancy in the whole population $(r=-0.190, p=0.20)$. Weight gain during the pregnancy and weight gain until gwk 24 correlated positively $(r=0.412, p=0.004)$. The symptom levels of depression and anxiety questionnaires varied from mild to moderate (Table 1). All the infants were born full term (gwks 37-42), except for one (gwk 36). No statistically significant correlations existed between the different bacterial taxa or Shannon index and depression or anxiety symptom scores.

\section{Bacterial characteristics}

Fecal microbiota of the subjects was dominated by Firmicutes (average over complete study population $53.3 \%$ ) and Bacteroidetes (45.9\%). Other phylum such as Proteobacteria, Tenericutes, Cyanobacteria, Actinobacteria, Lentisphaerae, Fusobacteria, and Verrucomicrobia were detected from the samples with proportions $<0.5 \%$ on average (Table 2). More detailed bacterial abundance data are attached in the Supplementary Table S1 (Supplementary Data are available online at www.liebertpub.com/jwh). The abundances of bacterial genera or phyla did not significantly correlate with either prepregnancy BMI or GWG. Furthermore, alpha and beta diversity were not related to the prepregnancy BMI or GWG.

\section{Hierarchical clustering}

Hierarchical clustering of the GM data by the relative abundancies of OTUs at the genus level was performed to elucidate the different subgroups in the population. Subjects were divided into two groups (group 1, $n=12$; group 2, $n=34$ ) based on the unsupervised hierarchical clustering. Group 1 was dominated by Gram-positive Firmicutes and group 2 by Gram-negative Bacteroidetes phylum $(p<0.005$ for both, Table 2). There were no significant differences in the alpha diversity indices between the two groups $(p>0.05$, Table 3). The Adonis statistics showed that groups were associated with unique GMC using weighted UniFrac analysis $\left(R^{2}=0.25, p=0.001\right.$, Fig. 1A), but not unweighted UniFrac analysis $\left(R^{2}=0.021, p=0.44\right)$.

The clinical characteristics of both the groups are presented in Table 1. Group 2 had a higher GWG $(p=0.025)$, but no other differences (maternal prepregnancy BMI or weight, the weight gain category, maternal age, seasonal division, or depression or anxiety symptoms) between the groups existed ( $p>0.05$ for all). There were no differences in the neonatal measures and outcomes between the groups ( $p>0.05$ for all). Maternal medical conditions were evenly distributed between the two groups.

\section{Firmicutes and Bacteroidetes-dominated groups}

Clustering by Firmicutes/Bacteroidetes ratio was performed to compare unsupervised hierarchical clustering groups and observed clinical differences. The Firmicutesdominated group $(n=28)$ had lower GWG than Bacteroidetes-dominated group $(n=18)$, the Bacteroidetes group having a higher mean GWG than the Firmicutes group (15.0 vs. $12.1 \mathrm{~kg}$, respectively, $p=0.023$ ); the effect was more prominent among normal-weight mothers (16.7 vs. $12.5 \mathrm{~kg}$, respectively, $p=0.0077$ ) (Table 1). The distribution of weight categories (normal, overweight, obese) was different between the groups $(p=0.028)$, the Firmicutes group having higher proportion of normal-weight mothers $(85.7 \%$ vs. $61.1 \%$ in Bacteroidetes group). The duration of gestation was longer in the Firmicutes group $(p=0.025)$, whereas other selected neonatal measures and outcomes or maternal characteristics were similarly distributed between the groups. Maternal medical conditions were evenly distributed among the groups (Table 1). The Shannon index $(p=0.0072)$ and the number of observed species $(p=0.0047)$ were higher in the Firmicutes group, whereas Chao1 did not differ between the groups (Table 2). The Adonis statistics showed that the groups were associated with unique GMC using unweighted $\left(R^{2}=0.066, p=0.002\right)$ and weighted UniFrac analysis $\left(R^{2}=0.41, p=0.001\right.$, Fig. 1B).

\section{Discussion}

The earlier findings concerning GMC in general populations cannot be directly applied to pregnant women as the physiology of various organ systems, including the gastrointestinal tract is different during pregnancy. ${ }^{45}$ Pregnancy is a turbulent physiological state with altered energy metabolism and GMC and diversity. ${ }^{26}$ To date, there are only a limited number of studies that have concentrated on GMC during pregnancy, and, in addition, how it is associated with GWG and maternal BMI. Furthermore, these studies have reported varying results, especially regarding the role of $B$. fragilis within the Bacteroidetes phylum on prepregnancy weight and GWG. $^{24,25}$ The current study offers new insight into this debatable topic.

In line with the earlier studies, the tradeoff between the Firmicutes and Bacteroidetes phyla was a main driving factor for the population variation in microbiota composition, as the individuals could be clustered into two distinct groups dominated either by Firmicutes or Bacteroidetes. The dominance of Bacteroidetes was associated with the elevated GWG, especially among mothers with normal weight before pregnancy.

Previously, the phylum Bacteroidetes has been considered as a microbiological trait that associates to the lean phenotype

Table 2. Alpha Diversity Indices According Groups

\begin{tabular}{|c|c|c|c|c|c|c|c|}
\hline Diversity & Mean & Group 1 & Group 2 & $\mathrm{p}$ & $\begin{array}{c}\text { Firmicutes } \\
\text { group }\end{array}$ & $\begin{array}{c}\text { Bacteroidetes } \\
\text { group }\end{array}$ & $\mathrm{p}$ \\
\hline Shannon index & 2.11 & 2.27 & 2.05 & 0.058 & 2.24 & 1.92 & 0.0072 \\
\hline Chao1 & 49.72 & 50.51 & 49.44 & 0.64 & 50.84 & 47.98 & 0.068 \\
\hline No. of observed species & 48.5 & 49.42 & 48.18 & 0.48 & 50.32 & 45.67 & 0.0047 \\
\hline
\end{tabular}

Group 1 and 2 formed by hierarchical clustering. 
Table 3. Mean Relative Abundances of Phyla According Groups

\begin{tabular}{|c|c|c|c|c|c|c|c|}
\hline Phylum & $\begin{array}{c}\text { Mean relative } \\
\text { abundance }\end{array}$ & Group 1 & Group 2 & $\mathrm{p}$ & $\begin{array}{l}\text { Firmicutes } \\
\text { group }\end{array}$ & $\begin{array}{l}\text { Bacteroidetes } \\
\text { group }\end{array}$ & $\mathrm{p}$ \\
\hline Actinobacteria & 0.11 & 0.14 & 0.10 & 0.8193 & 0.14 & 0.08 & 1.0000 \\
\hline Bacteroidetes & 45.90 & 28.72 & 51.96 & $>0.005$ & 34.91 & 63.00 & $>0.005$ \\
\hline Cyanobacteria & 0.02 & 0.01 & 0.02 & 0.7241 & 0.03 & 0.00 & 0.0499 \\
\hline Firmicutes & 53.32 & 70.20 & 47.36 & $>0.005$ & 64.08 & 36.57 & $>0.005$ \\
\hline Fusobacteria & 0.01 & 0.00 & 0.01 & 0.7241 & 0.01 & 0.01 & 0.7257 \\
\hline Lentisphaerae & 0.01 & 0.01 & 0.01 & 1.0000 & 0.01 & 0.00 & 0.2042 \\
\hline Proteobacteria & 0.31 & 0.28 & 0.32 & 0.9912 & 0.35 & 0.24 & 0.8865 \\
\hline Tenericutes & 0.27 & 0.54 & 0.18 & 0.7241 & 0.40 & 0.08 & 0.0366 \\
\hline Verrucomicrobia & 0.01 & 0.01 & 0.01 & 0.9461 & 0.01 & 0.00 & 0.4081 \\
\hline Other & 0.06 & 0.10 & 0.04 & 0.7241 & 0.08 & 0.02 & 0.4061 \\
\hline
\end{tabular}

Group 1 and 2 formed by hierarchical clustering.

and weight loss. ${ }^{16,18}$ However, contradictory results have been reported $^{46}$ and our study does not show associations between BMI and Bacteroidetes phylum. A study by Collado et al. reported that $B$. fragilis, within the Bacteroidetes phylum, was more abundant in overweight mothers-to-be in the first and third trimesters and further correlated with the GWG in the third trimester. ${ }^{25}$ However, in a study conducted by Santacruz et al., the proportion of B. fragilis in gwk 24 correlated with normal weight and normal GWG. ${ }^{24}$ Some of the discrepancies between the studies can be explained by the different sampling season and the characteristics of the participant population, such as variance in weight and geographical distribution. Furthermore, the results presented by the previous literature were conducted by either FCM-FISH or qPCR-based methods and thus describe species-level results. On the other hand, the current article describes the higher taxonomic levels.

Notably, the groups formed by the Firmicutes/Bacteroidetes ratio served a more straightforward clinical interpretation in terms of the maternal weight and GWG than the unsupervised grouping based on the overall genus-level composition. Additionally, the duration of gestation was lower in the Bacteroidetes-enriched group, whereas the clinical implication is more debatable as other neonatal measures and outcomes did not differ among the groups. Moreover, the Bacteroidetes-enriched group had a lower alpha diversity, which previously has been associated with overweight/obese phenotype and higher weight gain in the nonpregnant population. ${ }^{47}$ Additionally, Koren et al. reported a decrease in alpha diversity from first to third trimester, ${ }^{26}$ but this has not been replicated. ${ }^{48}$ Stanislawski et al. showed that postpartum alpha diversity was lower in obese/overweight mothers, but no association between GWG and alpha diversity was observed. ${ }^{49}$

In our study, the Illumina MiSeq sequencing approach was used to analyze the GMC. This offers a more holistic view of the microbial communities within the gut than the targeted approaches used in the previously mentioned studies conducted with pregnant women. ${ }^{24,25}$ Conclusively, more studies are needed to evaluate the role of GMC and especially members of the phylum Bacteroidetes on gestational health.

The seasons of the sampling and psychiatric symptoms were taken into account in statistical analyses as confounding factors. Seasonal variation might affect the GMC at least at the population level, ${ }^{38}$ and there is great co-occurrence of obesity and depressive symptoms. ${ }^{27}$ However, neither season nor psychiatric symptoms seemed to influence the GMC or clustering analyses, indicating that the season of sampling time or psychiatric symptoms do not affect the connection between GWG and GMC. Additionally, pregnancy-related conditions (pre-eclampsia, gestational diabetes, and gestational hypertension) were taken into account, as the microbiome of various sites, mainly vaginal microbiome, can affect major obstetric conditions. ${ }^{50}$ In the current study, however, the subjects with obstetric conditions did not seem to affect the clustering, and thus were included in the study. In addition, the prepregnancy state of GMC, which forms the baseline for our analysis, is unknown. Hence, this study is associative rather than causative. Longitudinal studies with larger study populations would be essential to reveal the relationship between GWG and GMC in pregnant populations. Additionally, the recording of the dietary intake was not possible to include in this study, which is a limitation. Despite these limitations, our study provides support for the

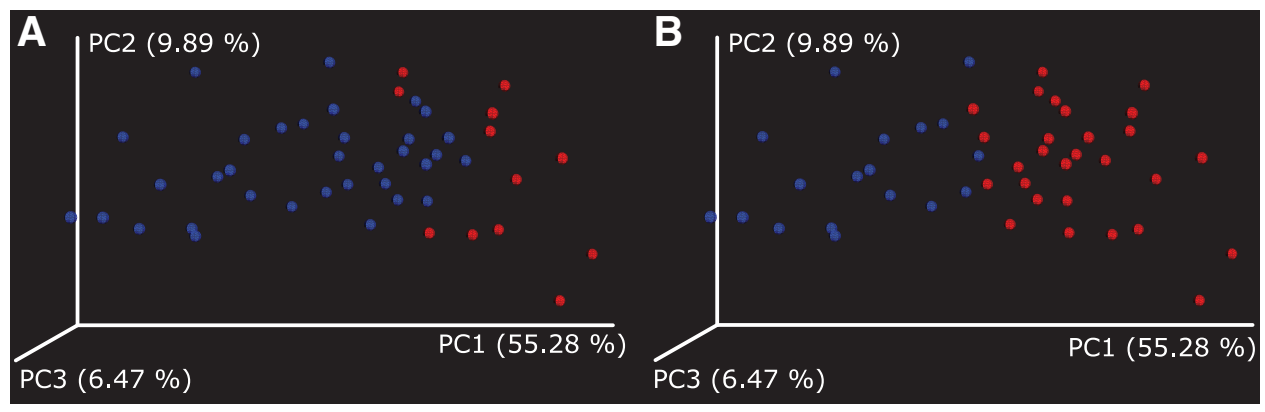

FIG. 1. Weighted UniFrac analyses according to (A) unsupervised hierarchical clustering $\left(R^{2}=0.25\right.$, $p=0.001)$ and (B) Firmicutes/Bacteroidetesdominated groups $\left(R^{2}=0.41\right.$, $p=0.001)$. 
hypothesis of a connection between GMC and weight gain in pregnant populations.

\section{Conclusion}

Excessive GWG was associated with distinct GMC in midpregnancy, with Bacteroidetes being prominent and Firmicutes being less prominent in the GMC among those with increased GWG. Moreover, reduced alpha diversity was observed among the Bacteroidetes-dominated subjects. Longitudinal research is needed to further illustrate the relationship between Bacteroidetes and weight and GWG in pregnant populations and to show how this affects infant health.

\section{Acknowledgments}

This research is supported by the Academy of Finland (grants 308176/L.K., 134950, 253270, 264363/H.K., 295741/ L.L.), the Yrjö Jahnsson Foundation (L.K.), the Signe, Ane Gyllenberg Foundation (L.K., H.K.), and the State Grants for Clinical Research (ERVA, H.-M.U.). Heidi Isokääntä is acknowledged for excellent technical assistance.

\section{Authors' Contributions}

A.-K.A. planned the design, collected and processed the biological data, and drafted the first article version. H.-M.U. and E.M. planned the design, and drafted and revised the second version of the article. H.P. participated in the analysis of the data and the article revision. A.R. processed the biological data, participated in the data analyses, and the revision of the article. S.P. participated in the data processing, analyses, and article revision. E.E. participated in the article revision and provided the funding for the study. L.L. contributed to the bioinformatics analyses and revised the article. L.K. participated in the planning of the design and revision of the article. H.K. planned the study design, provided the funding for the study, and revised the article.

\section{Author Disclosure Statement}

None of the authors has conflicts of interest to declare.

\section{References}

1. National Institute for Health and Welfare, Finland (THL). Perinataalitilasto - synnyttäjät, synnytykset ja vastasyntyneet [Perinatal statistics - parturients, delivers and newborns 2015]. 1798-0887; Statical report. 2016. Available at: http://www .julkari.fi/handle/10024/131259 Accessed November 10, 2016.

2. Devlieger R, Benhalima K, Damm P, et al. Maternal obesity in Europe: Where do we stand and how to move forward?: A scientific paper commissioned by the European Board and College of Obstetrics and Gynaecology (EBCOG). Eur J Obstet Gynecol Reprod Biol 2016;201:203-208.

3. Nodine PM, Hastings-Tolsma M. Maternal obesity: Improving pregnancy outcomes. Mcn, Am J Matern Nurs 2012;37:110-115. DOI: 10.1097/nmc.0b013e3182430296.

4. Barbour LA, McCurdy CE, Hernandez TL, Kirwan JP, Catalano PM, Friedman JE. Cellular mechanisms for insulin resistance in normal pregnancy and gestational diabetes. Diabetes Care 2007;30(Suppl 2):S112-S119.

5. Gregor MF, Hotamisligil GS. Inflammatory mechanisms in obesity. Annu Rev Immunol 2011;29:415-445.
6. Ovesen P, Rasmussen S, Kesmodel U. Effect of prepregnancy maternal overweight and obesity on pregnancy outcome. Obstet Gynecol 2011;118(Pt 1):305-312.

7. Ruager-Martin R, Hyde MJ, Modi N. Maternal obesity and infant outcomes. Early Hum Dev 2010;86:715-722.

8. Reichman NE, Nepomnyaschy L. Maternal pre-pregnancy obesity and diagnosis of asthma in offspring at age 3 years. Matern Child Health J 2008;12:725-733.

9. Rodriguez A, Miettunen J, Henriksen TB, et al. Maternal adiposity prior to pregnancy is associated with ADHD symptoms in offspring: Evidence from three prospective pregnancy cohorts. Int J Obes (Lond) 2008;32:550-557.

10. Lau EY, Liu J, Archer E, McDonald SM, Liu J. Maternal weight gain in pregnancy and risk of obesity among offspring: A systematic review. J Obes 2014;2014:524939.

11. Sommer F, Bäckhed F. The gut microbiota-Masters of host development and physiology. Nat Rev Microbiol 2013;11:227-238.

12. Heiman ML, Greenway FL. A healthy gastrointestinal microbiome is dependent on dietary diversity. Mol Metab 2016;5:317-320.

13. Turnbaugh PJ, Hamady M, Yatsunenko T, et al. A core gut microbiome in obese and lean twins. Nature 2009;457:480484.

14. Qin J, Li R, Raes J, et al. A human gut microbial gene catalogue established by metagenomic sequencing. Nature 2010;464:59-65.

15. Zhernakova A, Kurilshikov A, Bonder MJ, et al. Populationbased metagenomics analysis reveals markers for gut microbiome composition and diversity. Science 2016;352: $565-569$

16. Turnbaugh PJ, Ley RE, Mahowald MA, Magrini V, Mardis ER, Gordon JI. An obesity-associated gut microbiome with increased capacity for energy harvest. Nature 2006;444: $1027-1031$.

17. Bäckhed F, Ding H, Wang T, et al. The gut microbiota as an environmental factor that regulates fat storage. Proc Natl Acad Sci U S A 2004;101:15718-15723.

18. Ley RE, Turnbaugh PJ, Klein S, Gordon JI. Microbial ecology: Human gut microbes associated with obesity. Nature 2006;444:1022-1023.

19. Cani PD, Delzenne NM, Amar J, Burcelin R. Role of gut microflora in the development of obesity and insulin resistance following high-fat diet feeding. Pathol Biol 2008; 56:305-309.

20. Tremaroli V, Karlsson F, Werling M, et al. Roux-en-Y gastric bypass and vertical banded gastroplasty induce long-term changes on the human gut microbiome contributing to fat mass regulation. Cell Metab 2015;22:228-238.

21. Turnbaugh PJ, Bäckhed F, Fulton L, Gordon JI. Diet-induced obesity is linked to marked but reversible alterations in the mouse distal gut microbiome. Cell Host Microbe 2008;3: 213-223.

22. Vrieze A, Van Nood E, Holleman F, et al. Transfer of intestinal microbiota from lean donors increases insulin sensitivity in individuals with metabolic syndrome. Gastroenterology 2012;143:913.e7-916.e7.

23. Patterson E, Ryan PM, Cryan JF, et al. Gut microbiota, obesity and diabetes. Postgrad Med J 2016;92:286-300. DOI: 10.1136/postgradmedj-2015-133285.

24. Santacruz A, Collado MC, García-Valdés L, et al. Gut microbiota composition is associated with body weight, weight gain and biochemical parameters in pregnant women. Br J Nutr 2010;104:83-92. 
25. Collado MC, Isolauri E, Laitinen K, Salminen S. Distinct composition of gut microbiota during pregnancy in overweight and normal-weight women. Am J Clin Nutr 2008; 88:894-899.

26. Koren O, Goodrich JK, Cullender TC, et al. Host remodeling of the gut microbiome and metabolic changes during pregnancy. Cell 2012;150:470-480.

27. Luppino FS, de Wit LM, Bouvy PF, et al. Overweight, obesity, and depression: A systematic review and metaanalysis of longitudinal studies. Arch Gen Psychiatry 2010; 67:220-229.

28. Lihavuuden yleisyys Suomessa-THL. Available at: www .thl.fi/fi/tutkimus-ja-asiantuntijatyo/hankkeet-ja-ohjelmat/ kansallinen-lihavuusohjelma-20122015/lihavuus-lukuina/ lihavuuden-yleisyys-suomessa Accessed February 10, 2017.

29. Pirkola SP, Isometsä E, Suvisaari J, et al. DSM-IV mood-, anxiety- and alcohol use disorders and their comorbidity in the Finnish general population. Soc Psychiatry Psychiatr Epidemiol 2005;40:1-10.

30. Zimmermann U, Kraus T, Himmerich H, Schuld A, Pollmächer T. Epidemiology, implications and mechanisms underlying drug-induced weight gain in psychiatric patients. J Psychiatr Res 2003;37:193-220.

31. Amare AT, Schubert KO, Klingler-Hoffmann M, CohenWoods S, Baune BT. The genetic overlap between mood disorders and cardiometabolic diseases: A systematic review of genome wide and candidate gene studies. Transl Psychiatry 2017;7:e1007.

32. Jiang H, Ling Z, Zhang Y, et al. Altered fecal microbiota composition in patients with major depressive disorder. Brain Behav Immun 2015;48:186-194.

33. Rasmussen KM, Yaktine AL; Institute of Medicine (US) and National Research Council (US) Committee to Reexamine IOM Pregnancy Weight Guidelines. Weight gain during pregnancy. National Academies Press; 2009. Available at: www.ncbi.nlm.nih.gov/pubmed/20669500 Accessed April $1,2016$.

34. Verdu EF, Galipeau HJ, Jabri B. Novel players in coeliac disease pathogenesis: Role of the gut microbiota. Nat Rev Gastroenterol Hepatol 2015;12:497-506.

35. Huizink AC, Delforterie MJ, Scheinin NM, Tolvanen M, Karlsson L, Karlsson H. Adaption of pregnancy anxiety questionnaire-revised for all pregnant women regardless of parity: PRAQ-R2. Arch Womens Ment Health 2016;19: 125-132.

36. Cox JL, Holden JM, Sagovsky R. Detection of postnatal depression. Development of the 10-item Edinburgh Postnatal Depression Scale. Br J Psychiatry 1987;150:782-786.

37. Holi MM, Sammallahti PR, Aalberg VA. A Finnish validation study of the SCL-90. Acta Psychiatr Scand 1998;97: 42-46.

38. Davenport ER, Mizrahi-Man O, Michelini K, Barreiro LB, Ober C, Gilad Y. Seasonal variation in human gut microbiome composition. PLoS One 2014;9:e90731. DOI: 10.1371/journal.pone.0090731.
39. Rintala A, Pietilä S, Munukka E, et al. Gut microbiota analysis results are highly dependent on the 16S rRNA gene target region, whereas the impact of DNA extraction is minor. J Biomol Tech 2017;28:19-30.

40. Salomäki-Myftari H, Vähätalo LH, Ailanen L, et al. Neuropeptide $\mathrm{Y}$ overexpressing female and male mice show divergent metabolic but not gut microbial responses to prenatal metformin exposure. PLoS One 2016;11: e0163805.

41. Kozich JJ, Westcott SL, Baxter NT, Highlander SK, Schloss PD. Development of a dual-index sequencing strategy and curation pipeline for analyzing amplicon sequence data on the MiSeq Illumina sequencing platform. Appl Environ Microbiol 2013;79:5112-5120.

42. R Core Team. R: A language and environment for statistical computing. 2017. Available at: www.r-project.org Accessed May 2, 2018.

43. Oksanen J, Blanchett FG, Friendly M, et al. vegan: Community ecology package. 2017. Available at: https://cran.rproject.org/package=vegan Accessed May 2, 2018.

44. Benjamini Y, Hochberg Y. Controlling the false discovery rate: A practical and powerful approach to multiple testing. J R Stat Soc Ser B 1995;57:289-300.

45. Astbury S, Mostyn A, Symonds ME, Bell RC. Nutrient availability, the microbiome, and intestinal transport during pregnancy. Appl Physiol Nutr Metab 2015;40:1100-1106.

46. Schwiertz A, Taras D, Schäfer K, et al. Microbiota and SCFA in lean and overweight healthy subjects. Obesity (Silver Spring) 2010;18:190-195.

47. Le Chatelier E, Nielsen T, Qin J, et al. Richness of human gut microbiome correlates with metabolic markers. Nature 2013;500:541-546.

48. DiGiulio DB, Callahan BJ, McMurdie PJ, et al. Temporal and spatial variation of the human microbiota during pregnancy. Proc Natl Acad Sci U S A 2015;112:201502875.

49. Stanislawski MA, Dabelea D, Wagner BD, Sontag MK, Lozupone CA, Eggesb $\varnothing$ M. Pre-pregnancy weight, gestational weight gain, and the gut microbiota of mothers and their infants. Microbiome 2017;5:113.

50. Solt I. The human microbiome and the great obstetrical syndromes: A new frontier in maternal-fetal medicine. Best Pract Res Clin Obstet Gynaecol 2015;29:165-175.

Address correspondence to:
Anna-Katariina Aatsinki, BM
The FinnBrain Birth Cohort Study
Turku Brain and Mind Center
Institute of Clinical Medicine
University of Turku
Lemminkäisenkatu 3 a, Teutori Building, 2nd Floor
20520 Turku
Finland

E-mail: ankaaa@utu.fi 